ISSN 2693-2504

\title{
Biological Reset and Chronic Glaucoma Healing
}

Journal of Bioscience \& Biomedical Engineering

Case Report

Dr. Massimo Lombardi

LombardiEyeClinic, "Center for Regenerative Informational Medicine"

*Correspondence authors

\section{Dr.Massimo Lombardi}

LombardiEyeClinic

"Center for Regenerative Informational Medicine" Italy

Submitted : 17 Oct 2021 ; Published : 8 Nov 2021

\begin{abstract}
The Author reports in this Article, how, after being affected by Chronic Glaucoma for 40 years, he could finally, through the consistency of the "Evidence Based Medicine", find the causes of the Sickness and a local and general Cure "Effective and often Decisive" against it, with specific Omotoxicological-Organotherapeutic Chemistries.
\end{abstract}

Summary: The Author reports with this Article how, after having suffered a Chronic Glaucoma Disease himself (for 40 years), he found an Effective and "often" Resolving Cure with Local and General Therapy against Chronic Glaucoma with Homotoxicological and Organotherapeutic Drugs.

Epidemiology: A rough estimate is that Glaucoma affects about 80,000,000 patients worldwide by calculating the number of patients followed by the various (and not all connected) "Anti-Glaucoma Centers"

In my opinion, this value indicated by the O.M.S. only the "Tip of the Iceberg" and does not take into account Asia and Africa where only a few centers are connected with O.M.S. and furthermore in the Westernized World it is likely that there are patients not followed by the Centers but by hospital' borrowed Eye' Doctors and / or private Eye Doctors without counting "all those who suffer from it" but have not yet reached a "specific diagnosis" these considerations lead to an "only assumed Estimate" for a total of 600,000,000 patients and for many researchers also this Estimate appears largely approximate.

Chronic Glaucoma: Disease characterized by an increase in Endo-Ocular Pressure that goes beyond the normal value of: $16 / 20 \mathrm{~mm} \mathrm{hg}=$ (mercury) up to extreme values of $60 / 80 \mathrm{~mm}$ hg.

RIGHT EYE: HIGH CHRONIC GLAUCOMA (SEVERE) WITH ENDO-OCULAR PRESSURE ABOVE 40/60 mm hg WITH CORNEAL OPACITY FOR SECONDARY EDEMA

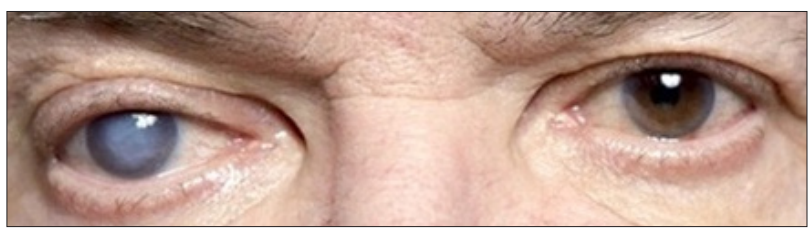

LEFT EYE: CHRONIC GLAUCOMA WITH MEDIUMHIGH PRESSURE WITH TRANSPARENT CORNEA

Collagen: body tissue, identical in basic structure throughout the body, present in ligaments, tendons, cartilage, synovium, Cornea, Sclera etc.

The Collagen Diseases are called: "Collagenopathies", can affect the whole body as in the "Lupus Eritematosus" or only one tissue as in "Keratoconus" =>the "Cornea" with an "Infectious Collagenopathy" caused by "Aspergillosis" which, I was able to Discover and Solve in 2002.

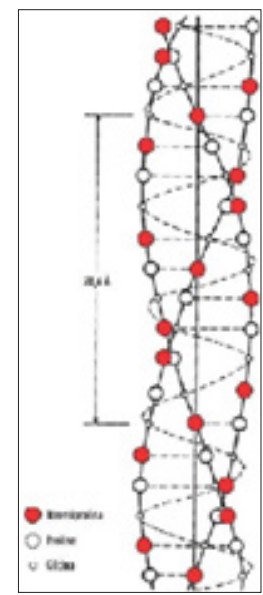

COLLAGEN STRUCTURE

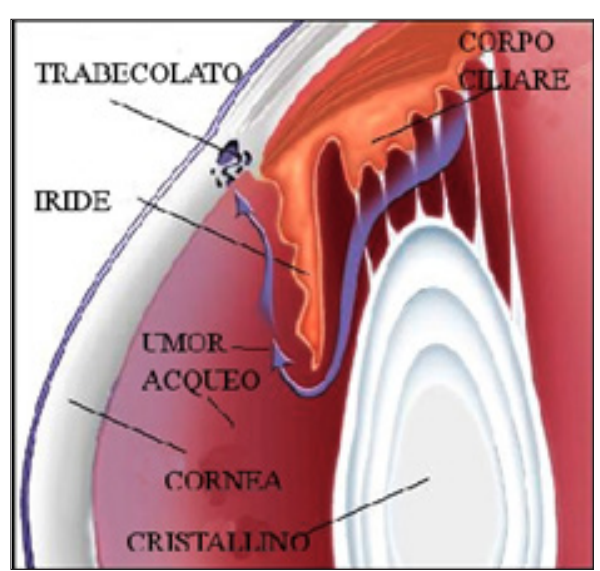

WATER HUMOR LEAKAGE THROUGH THE TRABECULATE WHERE ENDO-OCULAR PRESSURE IS REGULATED
Scleral Trabeculae: inside the circular channel called "Schlemm" that surrounds the base of the Cornea, in the inner part, on the periphery of the Cornea itself, made up of a Collagen network that serves as a "drain" of the "aqueous humor", produced by "Secretion" of the Ciliary Body, (a mixed structure), with the vascular part which borders on one side with the Iris (which gives color to the eye) and on the other 
with the spongy tissue, (a mixed structure), called !Uvea”.

Lamina Cribrosa: ( Cribrous-Foil ) a structure made of Collagen which, like a network, allows the "myelinated nerve fibers" of the Optic Nerve, the Blood and Lymphatic Vessels to pass through the empty spaces of the "net" in continuity with the Scleral texture precisely called "Sclera or Sclerotic" due to its compactness and biomechanical resistance that covers the whole Eye.

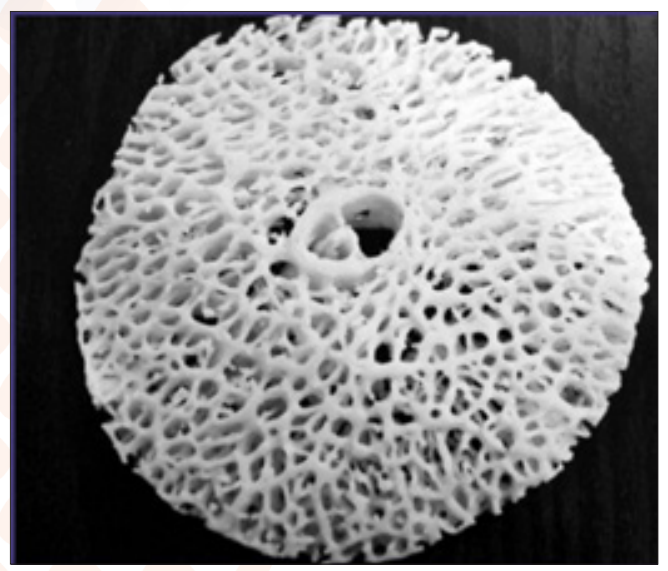

RETRO-OCULAR CRIBROUS FOIL = LAMINACRIBROSA

\section{Glossary}

Visual Field: instrumental examination aimed at determining a primary "visual function" which, while one Person stares at the center with one eye, makes see, at a "glance", even peripherally.

Sight: ability to read the "Optometric" table with letters / numbers to measure how many lines, or rather "Tenths" we can read at a distance of 3 , or, 5 meters, from: $1 / 10$ to $10 / 10$, with each eye. Meibonian and Zeis Glands regulate, in the Lid' border, tears and lubrication of the Conjunctiva.

Myelinated fibers of the optic nerve: are normal neuronal fibers covered, once outside the eye, by "a sheath of myelin" (like an insulating envelope) to cross, into the Optical Nerve, the brain.

Tachyphylaxis: phenomenon of "addiction to allopathic drugs" that lose their effectiveness even if with significant increase in number of administrations, because Patients become "use" to them.

Keratoconus: infectious collagenopathy secondary to Aspergillosis' Infection".

\section{Personal Clinical History}

I have suffered from Chronic Glaucoma since the age of 35 and I have tried all the therapies also with repeated sessions of "Argon-Laser iris' plastic" and with "Jag-Laser micro-punctures of Schlemm's channel", and the usual medical therapies with eye drops: Pilocarpine, Timolol and during my recent cataract surgery and IOL implantation with antiglaucomatous microsurgical sclerectomies in both eyes.

Having consequently reported severe worse changes in the O.C.T. of the left Eye and partial damage on the right Optic Nerve, seeing that, by now, after 40 years ( I am writing at 75 ) the situation was worsening faster, having also encountered the phenomenon of "Tachyphylaxis" with the antiglaucomatous eye drops, seeing an absolutely uncertain future for my eyesight.

I turned to the Most High, with a frank and net heart, and asked him for help.

The help came a moment later, when "suddenly" I doubted that this so serious Disease was not given to me as "a cross to bear to the end", but rather as a "profound stimulus", which "involved me personally", to make me find a "necessary Solution", as it is already happened with other diseases, discoveries and inventions that I had, previously, made with His Help, throughout my life since I was a 9 years old child and even before. (When I-have been presented as the "youngest inventor in Italy and Europe" in a specialized Italian National TV Program for Inventors, 1955)

\section{"Updated definition" of the- etiopathogenesis of Chronic Glaucoma}

Disease caused by a "high degree of chronic systemic inflammation" with "chronic accumulation of toxins" with consequence that these last lead to a "high activation of inflammatory cytokines". Located on the right side of the "Tissue Degeneration" of the "Neuro - Sensory - Ectoderm" in the

\section{"Recheweg Complete Table of Homotoxicosis".}

\section{Action' Mechanism}

The toxins generate a "Chronic Inflammation" of the "Collagen", and go to "Edemize" "first" and after with the Chronicization of the phenomenon, will "thicken" sclerosing the "Trabeculate of the Schlemm's Channel", (made of Collagen), making it narrower and reducing the room for the passage of the aqueous humor and also reducing consistently the normal drainage "flow rate" resulting in a progressive increase in the internal pressure of the eye: => Glaucoma. 


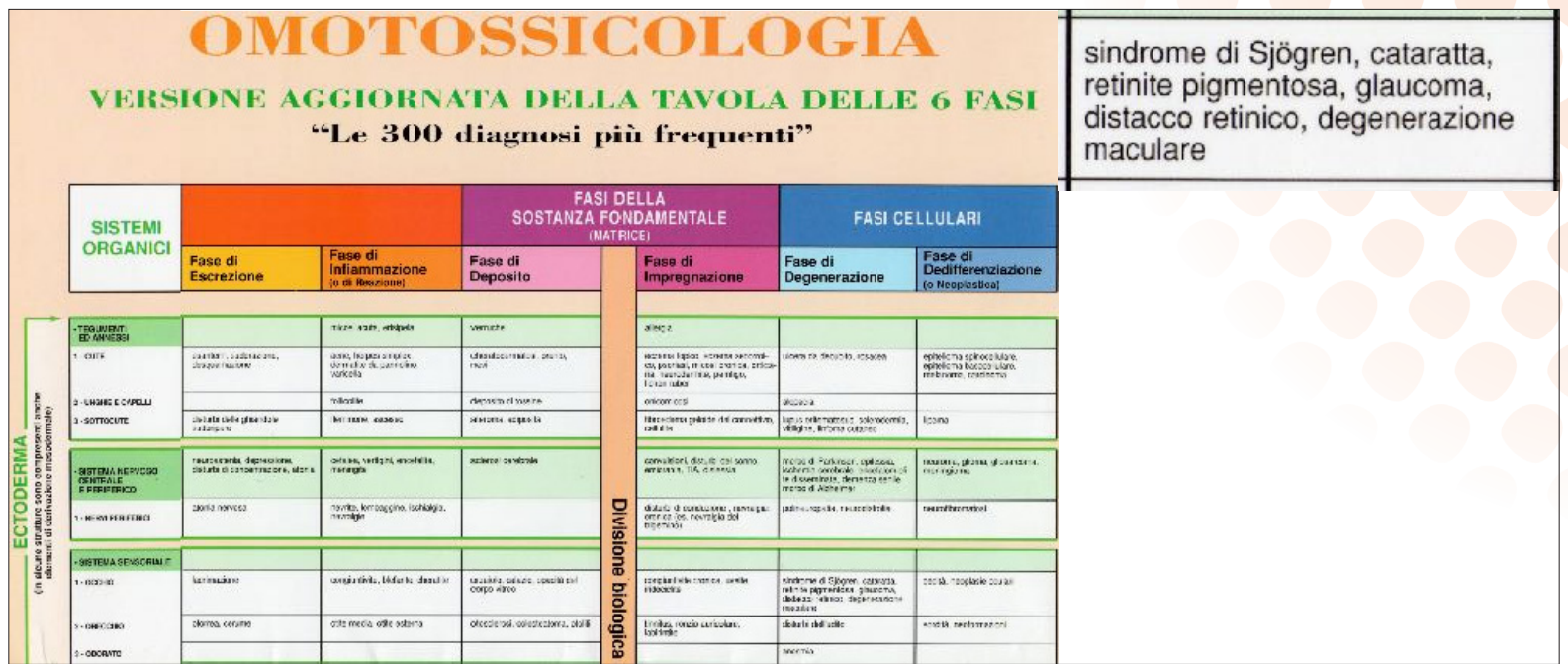

The "Corneal Trabeculate", where the outflow of aqueous humor occurs: is the "drainage pipe" of the same "aqueous humor - drainaging' system", made, as already explained above, by Collagen as well as the: "Scleral Lamina Cribrosa", network, through which pass the "myelinated fibers" of the Optic Nerve and the "Blood and Lymphatic Vessels", which, "progressively thickening", are "Strangling and Ischemizing" them. - "Chronic Glaucoma" can therefore be classified as a :

\section{-Collagenopathy by->Inflammatory-Chronic-Sclerosing-} Disease-

Treatment, in a modern "Holistic Vision" cannot, therefore, be only of the "Organ", as up to now happened with Allopathic Therapies, but rather of "System" where we must treat:

\# the whole "Collagen network system" both of the "Trabeculate in Schlemm's Canal" (where the initial outflow of aqueous mood occurs ) and of the "Lamina cribrosa" ( where the myelinated neuritic terminations fibers, Vessels and Lymphatics of the Optic Nerve pass through ) \#

\section{Materials and methods}

1. "General administration of "Homotoxicological and organotherapeutic anti-inflammatory drugs",

For specific draining, detoxifying and stimulating the "Cellular and Collagen' Tissue- Regeneration".

2. A certain number of Sub-Bulbar mini-injections with an insulin needle with same Chemistries to reach more easily the "Lamina Cribrosa" in the back k of the Eye and speed the "Healing". 3)The "local" administration of "Galenic Eye Drops", "conceived and successfully tested", with a "Cocktail of Homotoxicological drugs" which leads to a "slow but gradual improvement" of the "Endo-ocular Pressure" within 3-6 months by administering it 6 -10 times a day: during the $24 \mathrm{hs,} \mathrm{before} \mathrm{falling} \mathrm{asleep,} \mathrm{as}$ you get up during the night and in the morning as soon as you wake up. This local administration will lead, in its process of "progressive detoxification" and consequent "dis-inflammation", to a slow and progressive reduction up to the complete "normalization" of the thickness of the "networks" of the "Drainage Collagen System" reactivating a "regular outflow" and the maintenance of a "normal" or slightly "hypotonic" "Endo-Ocular Pressure" thus favoring a greater "Hematic-Turn-Over" with a "greater venous return", improving "Oxygenation" of the Retina, blood and lymphatic vessels and of the myelinated neuritic fibers of the Optic Nerve.

\section{Personal Note}

// This discovery is based on an "Intuition", inspired by the Most High, in a moment of profound consternation at the evidence of my progressive and unstoppable Visual deterioration, including the "Pharmacological Tachyphylaxis", which, after 40 years of Chronic Glaucoma, led me to ask Him for help.

The help was immediate, with the "intuition" and the immediate "consideration" that according to traditional: "Chinese Natural Medicine" there is a "Twinning between hollow (or empty) organs and full organs", therefore, "Eye" which is a "Full Organ" is "Twinned", by the meridian' connections of "Chinese Natural Medicine", with the "Stomach" which is a "Hollow Organ".Also in Traditional West Universities in Ophtalmology is known that Meibomians and Zeis Glands, into the Lid' Border are connected through the Lymphatic System as a Secondary Vicariant Drainage System for expelling surplus' toxins not metabolized through Intestine, in case of a substantial intoxication of the gastrointestinal system .

I understood in a moment that the Solution was to "Reverse the Paradigm" and treat "the Eye" as if I had to treat the "Inflamed Stomach", and therefore I understood how the Origin and Cause of Chronic Glaucoma was the consequence of a : ->Chronic-Inflammation->Sclerosis and Shrinkage of Collagen' Fibers into the Schlemm' Channel ( Main Drainage System ) and Lamina Cribrosa //

*You will realize at some point, after a few months of Therapy, that no longer should be needed all those daily administrations and that their number could be, "progressively", decreased, always keeping the Ocular Pressure "under control", as the "Detoxification", that it is the "Reset", proceeds and the Chronic Inflammation decreases, "the organism slowly regains" its "Balance" with the "normalization" of the "Eye' 
pressure" and an "effective complete oxygenation".

It must be pointed out that if the Patient - does not - "definitely change" many of his "Wrong eating habits and lifestyle" with the "only Eye' Drops administration" without a : =>

"Correct detoxification and normalization therapy of Chronic Inflammation" and therefore of the consequent reduction and possible arrest of the "Storm-Cytokines",=> it will not be "sufficient" to "normalize" the "Ethio - Pathogenetic Framework" in a "definitive way".

The persistence of "Pathogenic Noxa" does not allow for a "Real and Deep Detoxification" and therefore a true and lasting "Normalization of functions and systems" that means $=>$ "Healing".

Fortunately, even with an "incomplete" "Restituzio ad Integrum"=" Return to Integrity", which will not be able to reach a "Complete Biological Normalization", the "Stem Cells" will be "usefully" reactivated, because "wisely programmed" to be able to function even in the presence of "limited imperfections "of "Biological-Functional-Systems" to stop the disease that had "become chronic".

Therefore, if the Patient does not change "radically" his "Lifestyle" by correcting "many" but not " all" his "Wrong Nutritional Habits", only transitory improvements can be achieved, even if with a good improvement in the "Intraocular pressure" and "Sight", but he "Never" could reach one:

!Real Complete Healing" \#.

\# We must make sacrifices to defeat our Worst Enemy: The "Wrong-Habits" \#

\# We should also "know" how to eat "without harming ourselves", that is "not an easy task", nor, can be explained in this work, but I do explain all the right correct informations to my patients, because we live in a "Fake World and eat Fake Food" that it is "SURE" for every "affected" patient, and we have also many wrong Life-Styles that affect our lives too \#

\section{Disease' Behavior}

Is useful to remind that many Chronic Glaucoma suffer "most severe damage during the night":

Not being conscious, while sleeping, one usually does not notice the more or less slight soreness of the eyeballs due to the increased "Endo-Ocular" pressure, also because over time the "neurosensory pressure" terminals" "get used to the pain", depriving the patient of the most important "Alarm' Bell" to realize the "progressive worsening damage".

Because, often, we go to sleep after more or less abundant and / or toxic "Libations", and often, soon after dinner, we have not given the stomach the right time to digest "sufficiently" and therefore "Increasing the Toxic-Inflammatory Load" and with the release of the "toxins" a new "Cytokine Storm" develops and a new worsening due to the further "Thickening" and "Reactive -Inflammatory-Sclerosis" and then "Ischemizing" further the "Trabeculate of Schlemm's Canal" and the "Collagen network "Of the" Cribrosa Scleral Lamina "of the Optic Nerve through which the nerve fibers and vessels pass "Progressively Strangling and suffocating them" for an "Ischemic Anoxia" with the "progressive narrowing, from reactive thickening", of the Scleral' "Lamina Cribrosa" of the Optic Nerve. / In fact, even after having achieved the normalization with a successful Antiglaucomatous Surgery, and additionally a "Hypotension", of the Endo-Ocular Pressure, since "field and visual damage do not cease" indeed at contrary "continue to worsen" as, or, more slowly than before, "demonstrating" that in the Pathology is not "only" the "pressure increase of the Eye" that is the "Cause" of the progressive damage of the Nervous fibers, but, that there is also an "other Cause" equally and perhaps "more compromising" that causes a more Definitive Damage or "Terminal" Damage to the Retina, this also finally explains the "Progressive Damage" that "Mysteriously" hit patients affected by the "Low Pressure Glaucoma" where all the Damage is due to the : "Lamina Cribrosa Shrinkage of the Optical Nerve fibers and Blood and Lymphatic vessels". /

The increased Ocular Pressure directly causes the "crushing of the photoreceptors" and the "neuritic fibers of the Optic Nerve "Kneeling" on the Retina' Optic Nerve Head border" and then "strangling and ischemizing" them, "compressed" against the "rigid circular ridge" that is the "scleral' hole circle" where Optic Nerve fibers and Vessels pass through the Sclera opening like an "umbrella "into the Retina.

Photo of the Retina with the Head of the Optic Nerve like a "Funnel" Excavation and Optic Nerve Bleaching for Ischemia of Retinal Vessels that "Kneel" due to excessive Endo-Ocular Pressure on the compact circular ridge of the "Scleral Hole" where the "un-myelinated" neuritic fibers and vessels of the Optic Nerve pass through.

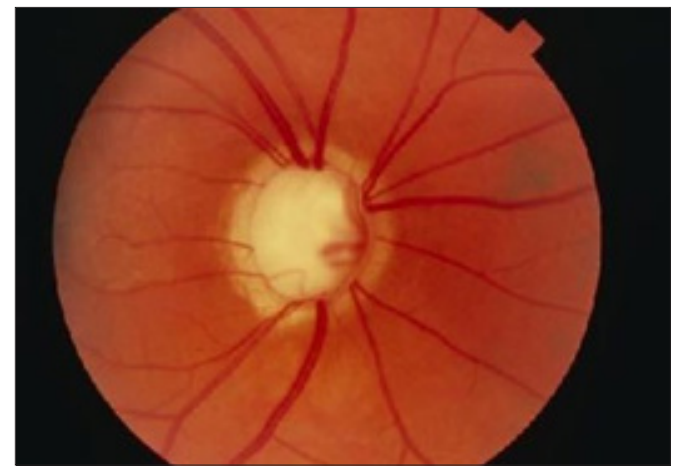

With the progressive damage of the: Sight, VIsual Field and Colors' vision up to their loss. While there is, at the same time, a progressive shrinkage, a "Strangulation" of all the "Collagen Networks" with the "Progressive-Toxic-ChronicInflammatory-Ischemic-Process" consequent to a "Edema" first and a "Sclerosis" after, with a progressive "Thickening" of the "Lamina' Cribrosa" = "Cribrous Foil" of the Optic Nerve which "progressively strangles" the "myelinated neuritic fibers" and the blood and lymphatic vessels of the Optic Nerve. 
Photo of the Optic Disc into the Retina, and the OCT of the same with a deep excavation of the Optic Nerve caused by High Ocular Pression.
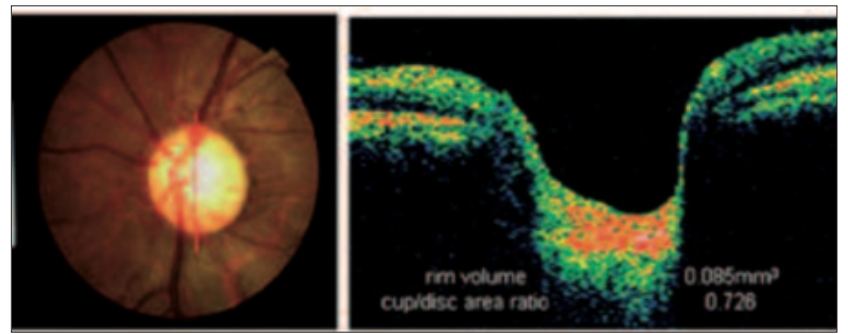

This explains, "conclusively", how and why the definitive damage to the neuritic fibers of the Optic Nerve can progress even after the primary "pressure problem" has been, "optimally", already resolved (with an intervention), with the "normalization" or even the !hypotension" of the Endo-Ocular Pressure as for the "Low Pressure Chronic Glaucoma affected Patients".

\section{Clinical cases, solved}

First case is the Author, who was able to live and see the darkness slowly but surely fall upon his eyes, before having found the "correct path to follow", and after having finally understood the "Causes of the Disease" and "How to cure it", who began to see the light and the contrast of the images improve, day after day, and the colors resume in a slow but progressive way as well as the "Vision" and "Visual Field" after the "normalization" of the Endo-Ocular Pressure and the "relaxation" of the nervous fibers and vessels into the "Lamina Cribrosa' framework".

The feeling of recovery and the awareness that the problem may have been solved is contemporary with the progress of the effectiveness of Local and General Therapy and can only well be understood by having experienced it in person, as happened to me, and this for the :

"Will of the Most High".

After the first improvement and the conscious progression of my Healing, I began to treat my patients with "identical Treatment" and "identical results", "all them improved" already with the local treatment with the "Galenic eye' drops" I designed and prepared and have been (cured and) healed all those who have had the "courage and constancy to change" their "unconscious" : "wrong lifestyle" and "nutritional habits" forever, after a profound process of Detoxification and Drainage "inducing" with my "Sub-Bulbar " ( using an insulin needle ) small Injections of 2.5 c.c. an already proved : => "Regeneration-of-Retinal-and-Optic-Nerve-Stem-Cells".

\section{Summary}

The Bio-mechanism of the Optic Nerve fibers "Strangulation" is then caused as a consequence of the "Chronic inflammation" consequence of the "Chronic Intoxication" of the - Gastrointestinal apparatus -, first by the "increased intraocular pressure" provoking the Crushing with Kneeling on the scleral border of the hole that allows the passage of the Optical Nerve through the "Sclerotic" and finally with the "tighteningstrangling-compression" of the nervous fibers, blood and lymphatic -vessels of the Optical Nerve passing through the "Lamina Cribrosa" Collagen sponge.

\section{Conclusions}

The therapy I experienced, while it generates a progressive reduction of the chronic reactive inflammatory thickening of Collagen structures, oxygenating the same structures and Retinal Photoreceptors, un-myelinated neuritic fibers (first in the intra-ocular tract and then in the tract that crosses the "Lamina Cribrosa" and out of the eye into the optic nerve) and finally all the "Retinal blood and lymphatic vessels " promoting a : "Self-healing" from "Biological Reset"obtained by "spontaneous reactivation" of "Autologous Stem Cells" which finally, with the "substantial reduction of circulating inflammatory toxins" that had induced an "abnormal" and "chronic Cytokines activation", they can resume their "natural and spontaneous ability to repair biological damage" of any kind, since they had been "deactivated" by the absence of a sufficient "biological balance" for the "Chronic-Reactivity-toCytokines-Waterfall" that causes a "block" or "strongly inhibit and reduces" the "Activity and Spontaneous Regeneration of the Stem Cells" [1-4],

which "have been "designed" to undergo "ACUTE or SHORT “ but " NOT - PERSISTENT “ "inflammatory processes”, therefore the "PERSISTENCY of CYTOKINS' WATERFALL " that "INHIBITS" the "automatic reparative response" of the same STEM CELLS who "NO LONGER RECOGNIZE" the "Original Biological Program", with which they had been "created and programmed", now in this "Pathological Situation" are confused, inhibited and disoriented by the "CHRONICIZATION" of !CYTOKINE' STORM" that "INACTIVATES their FUNCTION' PROGRAM".

\section{Final summary}

My Therapy "Reset first and then Reprogram" the Autologous Stem Cells, having "substantially reduced the excess of circulating cytokines" which prevented their "spontaneous reactivation".

Please Note: By "Reprogramming" of Stem Cells I mean the possibility of bringing the same Stem Cells "as close as possible" to the "Original Programming of the "man / woman" Cyborg" (which is formed by a Softer and a Hardware as a "very advanced Biological Computer") performed with the "Initial Environmental Biological Programming' conditions" when happened : the "Act of the Creation of Man and Woman" in the Eden. So with the understanding of the "Principles of Correct Biological Functioning" we can: "Correctly Cure and often Heal" Diseases.

Much of what I discovered it is also thanks to the "Teaching" of my "Grand-Masters" whom God in his Great Generosity made me meet to make me walk the path He designed for me and that I consider "Spiritual Co-Authors" of my Discoveries 
and of my Scientific Articles reason why I am remembering their Names and Professional Positions here.

\section{The Late:}

Prof. Aldo Spirito, Biologist University' Lecturer in Rome, proposed for the Nobel Prize in Science. Prof. Giovanni Battista Bietti, President of the O.M.S. for Ophthalmology, Professor in Rome.

Prof. Svyatoslav Nicolajevich Fyodorov, Academyc of Sciences of Russia, Director of the "Moscow' Eye MicroSurgery Center".

Prof. Orfilio Pelaez, Director of the Center for the Treatment of "Retinitis Pigmentosa" :

"Camillo Cienfuegos" Havana, Cuba, "creator" of the "Eye Re-Vascularization", in fact, "He" has been the "Unconscious Precursor" of the "Regeneration of Ocular Stem Cells".

And a $5^{\text {th }}$ "Grand Master": Prof. Leonello Milani, President of the "International Academy of Physiological Regulatory Medicine" that with his "Master's Lessons" made me grow in Scientific Knowledge, allowing me to reach my latest discoveries in Homotoxicology.

Below is a List of Discoveries and Inventions, of the Author, in the "Medical Field", to make better understand that "nothing happens by chance" and that the true Scientist is a "Researcher" aimed to understand, with "Net Mind and Spirit", the "Principles", first and then the "Mechanisms", of Nature, often helped, in various ways, from the Most High.

1. The Correction of "High Myopias" with a "Diamond MicroScalpel" (free hand): with the R.K. ="Radial Keratotomy" that allowed with a program "devised" by me to correct up to - 21 diopters, having passed all the programs of a "Data and Program Computerized Processing Center in Prof. Fyodorov' Institute", in Moscow, with a "ruler, pencil and graph-paper", 1982.

2. "Cryogenic Treatment" on the Cornea with the "Cryo" (cryogenic probe for the "intracapsular extraction of the Cataract" which cools down to - 70 degrees cent.) in Herpes Simplex "relapsing keratitis", with their definitive "Healing", preserving the Cornea Transparency, 1982.

3. the !Immuno-modulating Medical Therapy" which stops the Progressive Myopia caused by a group of "NeuroAnisotropic Viruses" which prevent, "delaying" it, to terminate the "genetic program" of "elongation of the Eyeball" from "intrauterine waist" to the "normal length of $23 \mathrm{~mm}$ (front-back measurement of the Eye-bulb) of the Adult "with a "6 months" Medical Therapy, 1982.

4. First "closed" cataract surgery, in high myopic, "Without Cut and Suture" with 2 separated entrances for the needles: one "aspirating needle", (including the "safe" cleaning of the capsule), split from a second one for the "infusion" each one of $1 \mathrm{~mm}$ in diameter, 1983.

5. Realization of a "coaxial-needle" for Cataract Operation, of $1.2 \mathrm{~mm}$ in diameter, 1 "aspirating", with an automatic valve, (designed by me), with a pre-calibrated silicone vent tube ( in order not to exceed the breaking pressure limit of the capsule ), and 1 coaxial to first "irrigating", mounted on a "disposable"( plastic) syringe, that did same work as a "Kelman's Phaco computer-machine" (but a bit "cheaper": 1,000 lire against 70,000,000 lire) and without need of electricity, thus allowing "advanced intervention" even in emergency environmental conditions, 1984.

6. the A.R.K. (Asymmetric Radial Keratotomy) technique used today by me as "Mini-A.R.K.", to Obtain a "Corneal Geometrical Implosion" with a first result to "Re-Increase" central-paracentral "Corneal thickness", (thinned due to Keratoconus) and second the "simultaneous correction" of the "Secondary Refractive Defect" as Astigmatism and / or / Myopia or both, resulting from the Keratoconus' Corneal Curvature Ectasis Pejorative Modification, 1984.

7. The first surgery of "congenital cataract" in "extracapsular + IOL-Sputnik implant", with the needle, (which I invented), in an 8-month-old child in the Ophthalmology Department of the Colombo Hospital in Sri Lanka, 1985.

8. LASIK (Femtosecond-Laser surgery) a Technique that I conceived and tested first on Pig' Eyes with the Prof.' Dragger' Microcheratome, in 1989.

9. Discovery of "Aspergillosis" as the "true cause of Keratoconus" that it is, in fact, a "Corneal Collagen Infection" = "Collagenopathy Secondary to Fungal Infection" with strong weakening and restructuring of the "Collagen Fibrils" that with the application of the "BioElectronic-Resolutive-Therapy" reaching therefore the "Complete Resolution" of the Disease, (no more "stupid Theories" with the involvement of "phantom genetic Diseases"), 2002.

10. First "Autologous Stem Cell Transplants" in the "Eduardus KrankenHaus" Hospital in Cologne, Germany, with the authorization and supervision of the German Ministry of Health, February 2008.

11. Subsequent Discovery of "Regenerative Informational Medicine" for "Autologous Stem Cells", without the need of a "direct transplantation" of same Stem Cells, end of 2008.

12. Definition of "Biological Life and Biological Information" and of "True Nature of Water'Memory" a "debated topic" but "never discovered" even by Eminent Nobel Prices (Benveniste, Pop, Mantagnier and others) with my "Discovery" of "Ice-Micro-Crystals" in blood and intra and extra-cellular body fluids, which !preserve the Memory of Biological Programs imprinted on the "Ice Cristal' Symmetrical Planes" as a "Sylicon-Micro-Chip" throughout the body, January 2009.

13. Recent Discovery of the "Biological Reset with Healing of Chronic Glaucoma" with an appropriate and Effective Local and General Therapy, January 2021. 


\section{References}

1. Dr. Massimo Lombardi (2018) "Retinal Stem Cells Reprogramming" - Journal of Ophtalmology \& Clinical Research, ISSN: 2573-9573.

2. Dr. Massimo Lombardi (2018) "Retinal Stem Cells Reprogramming Using Omotoxicological Pharmaceuticals Blend Therapy" through Retrobulbar Injections,-Journal of Stem Cell Research.

3. Dr. Massimo Lombardi (2021) "Retinal and Body 'Stem Cells Reprogramming" - Acta Scientific Ophtalmology ISSN: 2582-3191.

4. Dr. Massimo Lombardi (2018) "Keratoconus Etiopathogenesis and True Cure, Modern Concepts" Journal of Clinical Review \& Case Reports, ISSN: 25739565.

Copyright: C2021 Dr.Massimo Lombardi. This is an open-access article distributed under the terms of the Creative Commons Attribution License, which permits unrestricted use, distribution, and reproduction in anymedium, provided the original author and source are credited. 